\title{
Impaired lipid clearance in patients with previous acute pancreatitis
}

\author{
S GUZMÁN, F NERVI, O LLANOS, P LEÓN, AND V VALDIVIESO \\ From the Departimento de Gastroenterología, Escuela de Medicina, Pontificia Universida Católica de Chile, \\ Santiago, Chile
}

SUMMARY Fasting serum triglycerides were measured in 52 patients who had sustained an attack of pancreatitis (gall stone related 33, alcoholism six) at least six months earlier. Several patients $(23 \%)$ had raised fasting serum triglycerides, with a type IV phenotype in all but one patient. The 40 patients with normal fasting serum triglycerides received an oral load of $100 \mathrm{~g}$ sunflower oil to compare their clearance of dietary triglycerides with that of a control group of 54 subjects. The clearance of ingested triglycerides was significantly impaired in the patients - irrespective of the presumed aetiological factor, or clinical conditon associated with pancreatitis - compared with the clearance in controls. A triglyceride tolerance test is the only way to detect those patients in whom a future attack of pancreatitis may be precipitated by a diet rich in fat, or endogenous over production of triglycerides as after an alcoholic debauch.

Several lines of evidence suggest an association between high serum triglyceride levels and pancreatitis. ${ }^{1-5}$ Thus, the occurrence of pancreatitis in the rare congenital conditions of primary lipoprotein lipase ${ }^{6}$ or C-II apoprotein deficiency ${ }^{3}$ is well established, while raised serum triglycerides are frequently noted in patients with alcohol related pancreatitis. ${ }^{4}$ Exactly how hypertriglyceridaemia precipitates pancreatitis is unknown, but studies in the isolated canine pancreas suggest that pancreatitis may be due to toxic fatty acids generated within the gland by the action of lipase on triglycerides in pancreatic capillaries. $^{7}$

Considering that in Chile acute pancreatitis associated with gall stones is more common than alcoholic pancreatitis, ${ }^{8}$ we decided to carry out an investigation to see if patients who had suffered an episode of acute pancreatitis could have abnormalities of triglyceride metabolism not detectable in fasting conditions. For this purpose fasting and postprandial serum triglyceride levels were measured in patients with previous acute pancreatitis, regardless of the presence of other potentially etiologic factors, such as gall stones, and the results were compared with values obtained in a group of control subjects.

Address for correspondence: Dr Sergio Guzmán, Departamento de Gastroenterología, Pontificia Universidad Católica de Chile, Casilla 114-D, Santiago, Chile.

Received for publication 29 October 1984

\section{Methods}

\section{PATIENTS}

A prospective study was conducted in 52 patients admitted to the Hospital Clínico de la Pontificia Universidad Católica de Chile between 1976 and 1982. The diagnosis of pancreatitis was based on the presence of compatible clinical signs and symptoms plus blood levels of amylase greater than or equal to 250 Caraway units and 24 hour urinary amylase higher than 5000 Caraway units (20 patients). In 32 patients the diagnosis was confirmed by surgery.

Fasting serum triglyceride determination and lipoprotein electrophoresis were carried out at least six months after the patients had clinically recovered. The upper limit for normal serum triglycerides was considered to be $1.70 \mathrm{mmol} / \mathrm{l}$. In those patients with normal fasting serum triglycerides and normal electrophoretic lipoprotein phenotype, an oral load of $100 \mathrm{~g}$ of fat (sunflower oil) was given after an overnight fast. Serum triglyceride concentrations were measured before and $3,6,9,12$, and 24 hours after the oral load. Throughout the test the patients were kept on a fat free 500 calorie liquid diet. The results obtained in the patients with previous pancreatitis (study group) were compared with those obtained in 54 subjects without previous pancreatitis (control group), which included healthy volunteers and patients hospitalised for elective surgery for gall stones or hernias. Studies undertaken in the control group also included measure- 
ments of fasting serum triglycerides and glucose and lipoprotein electrophoresis. The sunflower oil oral load test was done in all these subjects, independently of the fasting electrophoretic phenotype. The age sex distribution and the frequency of diabetes, heavy alcohol consumption (more than $100 \mathrm{~g}$ per day) and biliary tract lithiasis of both groups are shown in the Table. Among the study group patients with biliary lithiasis, 11 had calculi in the bile duct, 10 had acute inflammation of the gall bladder (certified by histopathological study) and 12 chronic cholecystitis. At the time the study was undertaken, 11 of the study subjects were receiving a normal diet because their pancreatitis had been treated more than one year earlier. None of the patients had symptoms of chronic pancreatitis, but endoscopic pancreatography and exocrine function tests were not done to exclude this possibility.

Patients were considered to be diabetic when hyperglycaemia persisted after the acute pancreatitis episode had subsided. Serum triglyceride concentrations were measured using Carlson's $\operatorname{method}^{9}$ and lipoprotein electrophoresis as described by Chin and Blankenhorn. ${ }^{10}$ Frederickson's criteria ${ }^{10}$ was used to determine the lipoprotein electrophoretic phenotype. Informed consent was obtained from all subjects.

Intergroup differences in mean values were tested for statistical significance using Student's $t$ test or the $\chi^{2}$ test as necessary.

\section{Results}

In fasting conditions, 12 study patients had raised serum triglycerides, with a type IV electrophoretic phenotype in 11 and a type $\mathrm{V}$ pattern in the remaining subject. Of the 12 patients in the study group with an abnormal phenotype, only one was diabetic and none were known alcoholics. In the control group only one subject had raised fasting serum triglycerides with a type IV phenotype $(<0.05)$.

Table Clinical data in patients with pancreatitis (study group) and in subjects of the control group

\begin{tabular}{lll}
\hline & Study group & Control group \\
\hline No & 52 & 54 \\
$\bar{x}$ Age & $44(25-72)$ & $40(19-64)$ \\
Men & 34 & 33 \\
Women & 18 & 21 \\
Diabetes & 9 & 0 \\
Alcoholism & 6 & 0 \\
Biliary lithiasis & 33 & 18 \\
Estrogen treatment & 1 & 0 \\
Obesity & 0 & 2 \\
\hline
\end{tabular}

After the ingestion of $100 \mathrm{~g}$ sunflower oil, serum triglyceride concentrations in the study group patients were significantly higher than the controls at all times after the fat meal, except at 24 hours (Fig. 1). For example, at six hours mean values of the study group were $2.17 \mathrm{mmol} / \mathrm{l}$, whereas the equivalent in the control group reached only 1.24 $\mathrm{mmol} / \mathrm{l}$. In fact, at this time only four patients of the study group had triglyceride concentrations below $1.70 \mathrm{mmol} / \mathrm{l}$, whereas only five subjects of the control group had serum triglycerides above this value. When diabetic and alcoholic patients were excluded from the study group (eight diabetic, including two alcoholics and four non-diabetic alcoholics) (Fig. 1), the results were virtually identical to those seen in the whole study group, and the differences with the control group were equally significant.

Because the study group included more patients with biliary lithiasis than the control group, we compared separately the results in the population with and without lithiasis, for both the study and the control group. As shown in Figure 2, no differences were found between the subjects with and without gall stones. Similarly, there were no differences determined either by sex or age.

\section{Discussion}

The results of our work confirm previous reports that a high proportion of patients have raised serum triglycerides long after they have recovered from pancreatitis. ${ }^{11}$ We found this abnormality in 12 out of 52 patients $(23 \%)$ and all but one had a type IV

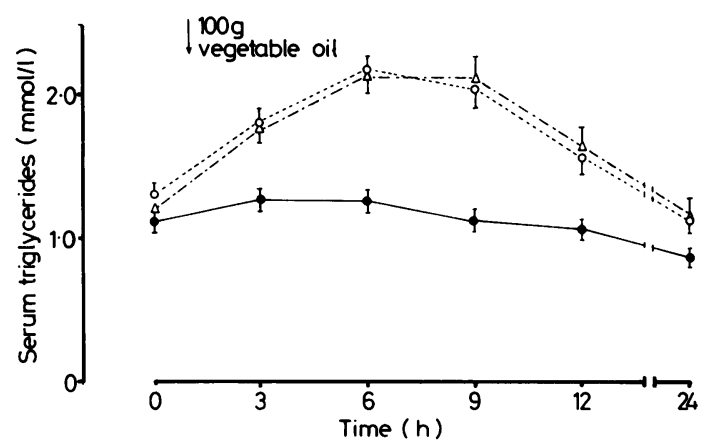

Fig. 1 Serum triglyceride levels (mmolll, mean $\pm 1 S E$ ) after an oral intake of $100 \mathrm{~g}$ of vegetable oil in the control group (-), in the study group $(\bigcirc \ldots . . . . . . .$. and in the study group excluding diabetic and alcoholic patients $(\triangle \longrightarrow \cdot \triangle)$. Differences between the study group and the control group were significant at 3, 6, 9 and 12 hours after the intake of fat $(p<0 \cdot 01)$. 


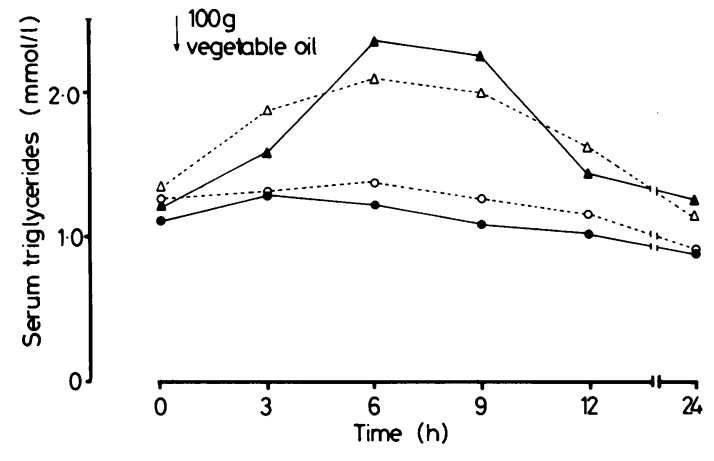

Fig. 2 Serum triglyceride levels (mmol/l), mean) after an oral intake of $100 \mathrm{~g}$ of vegetable oil in the study group and in the control group, plotting separately subjects with and without gall stones. Control group: without gall stones:

$(n=36)$; with gall stones: $\bigcirc \cdots \cdots \cdots \cdots(n=18)$. Study group: without gall stones $\mathbf{\Lambda} \mathbf{\Delta}(n=11) ;$ with gall stones: $\triangle \cdots \cdots \cdots \cdots \cdot(n=29)$.

electrophoretic pattern. Cameron et al ${ }^{12}$ reported that during the disease 18 out of 48 patients $(38 \%)$ had raised serum triglycerides and Farmer et ll $^{13}$ found this abnormality in 10 out of 45 patients (22\%): at the onset of the disease, most patients presented either a type I or $\mathrm{V}$ electrophoretic pattern, but after recovery most of those with persistently raised serum triglycerides exhibited a type IV phenotype. ${ }^{11}$

Our results show furthermore, that the clearance of an ingested fat load (100 g sunflower oil) remains impaired in several patients who had suffered an attack of pancreatitis, although their fasting serum lipids are normal. This defect is independent of the presence of diabetes and alcoholism, and was also present in patients with pancreatitis associated to biliary tract lithiasis. The detection of this abnormality, which may have implications in the dietary management of these patients, was only made apparent by a fat tolerance test. A similar finding, but in patients with high fasting serum triglycerides, was reported by Cameron et al. ${ }^{11}$ They suggested that this alteration could be important in the pathogenesis of the disease. This concept is supported by subsequent reports of pancreatitis caused by hyperlipemia. $^{35}$

Whether the abnormality in triglyceride metabolism precedes or follows acute pancreatitis cannot be answered by the present study. Experimental evidence in dogs suggests that pancreatitis can produce a rise of serum triglycerides, ${ }^{2}$ but a permanent alteration of lipid metabolism secondary to pancreatitis has not been shown. In man, Kessler $e t$ $a l^{1}$ described abnormal lipoprotein-triglyceride complexes resistant to lipoprotein lipase in one patient with pancreatitis and hyperlipemia. In another patient they found circulating inhibitors of lipoprotein lipase. ${ }^{14}$ These findings suggest that several factors could be involved in the development of abnormalities in the serum triglyceride concentrations during, and perhaps also after, acute pancreatitis, including a deficiency in the lipoprotein lipase system, apoprotein deficiencies or abnormal lipoprotein receptors.

The persistence of postprandial raised serum triglycerides for a long period of time after the episode of acute pancreatitis (at least six months in our study) suggest a relatively common and preexistent defect in lipid metabolism. It has been proposed that abnormally high serum triglycerides could be involved in the pathogenesis of acute pancreatitis by provoking high pancreatic concentrations of free fatty acids after the action of pancreatic lipase. Schmitz-Moorman and Böger ${ }^{15}$ showed that olive oil may cause liver, kidney, pancreatic or muscular necrosis if injected with pancreatic lipase. Saharia and coworkers ${ }^{7}$ showed that both triglycerides and free fatty acids were toxic in the isolated perfused canine pancreas. In addition, experimental data obtained in dogs, indicate that pancreatitis induced by intraductal injection of bile and trypsin was more severe in animals fed a high fat diet, than those fed a high protein or a high carbohydrate diet. $^{16}$

If the impaired clearance of serum triglycerides described in the present work proves to be a common and preexistent disorder in patients with acute pancreatitis, it may be hypothesised that a significant proportion of cases of pancreatitis are caused by an interaction of pancreatic lipase and triglycerides present in the capillaries of the pancreas. This abnormality would become significant in the pathogenesis of pancreatitis when the affected subjects are challenged with a high fat diet, estrogens or heavy alcohol consumption.

\section{References}

1 Kessler JI, Miller M, Barza M, Mishkin S. Hyperlipemia in acute pancreatitis. Am J Med 1976; 42: 968-76.

2 Wang C, Trauss L, Adlesberg D. Experimental pancreatitis and plasma lipids. Gastroenterology 1958; 35: 465-72.

3 Breckenridge WC, Little JA, Steiner G et al. Hypertriglyceridemia associated with deficiency of apolipoprotein C-II. $N$ Engl J Med 1978; 298: 1265-73.

4 Cameron JL, Zuidema GD, Margolis S. A pathogene- 
sis for alcoholic pancreatitis. Surgery 1975; 77: 754-63.

5 Weinberg RB, Sitrin MD, Adkins GM, Lin CC. Treatment of hyperlipidemic pancreatitis in pregnancy with total parenteral nutrition. Gastroenterology 1982; 83: $1300-5$.

6 Cameron JL, Crisler C, Margolis S, De Meester TR, Zuidema GD. Acute pancreatitis with hyperlipemia. Surgery 1971; 70: 53-61.

7 Saharia P, Margolis S, Zuidema GD, Cameron JL. Acute pancreatitis with hyperlipemia: Studies with an isolated perfused canine pancreas. Surgery 1977; 82: 60-7.

8 Puente S, Lasagna R, Sommariva J. Pancreatitis aguda e índices de gravedad. Rev Chil Cir 1979; 31: 183-90.

9 Carlson LA. Determination of serum triglycerides. $J$ Atheros Res 1963; 3: 334-36.

10 Chin HP, Blankenhorn DH. Separation and quantitative analysis of serum lipoproteins by means of electrophoresis on cellulose acetate. Clin Chim Acta 1968;
20: 305-14.

11 Cameron JL, Capuzzi GD, Margolis S. Acute pancreatitis with hyperlipemia. Evidence for a persistent defect in lipid metabolism. Am J Med 1974; 56: 482-7.

12 Cameron JL, Capuzzi DM, Zuidema GD, Margolis S. Acute pancreatitis with hyperlipemia: the incidence of lipid abnormalities in acute pancreatitis. Ann Surg 1973; 177: 483-9.

13 Farmer RG, Winkelman E, Brown HB, Lewis LA. Hyperlipoproteinemia and pancreatitis. Am J Med 1973; 54: 161-5.

14 Kessler JI, Kniffen JC, Janowitz HD. Lipoprotein lipase inhibition in the hyperlipemia of acute alcoholic pancreatitis. $N$ Engl J Med 1963; 269: 943-8.

15 Schmitz Moormann P, Böger A. Tissue damage by fatty acids released by lipolysis. Pathol Res Pract 1981; 171: 303-13.

16 Haig BTH. Experimental pancreatitis intensified by a high fat diet. Surg Gynecol Obstet 1970; 131: 914-8. 